\title{
ON THE MATHEMATICAL ANALYSIS AND OPTIMIZATION OF CHEMICAL VAPOR INFILTRATION IN MATERIALS SCIENCE*,**
}

\author{
Adi Ditkowski ${ }^{1}$, David Gottlieb ${ }^{1}$ And Brian W. Sheldon ${ }^{2}$
}

\begin{abstract}
In this paper we present an analysis of the partial differential equations that describe the Chemical Vapor Infiltration (CVI) processes. The mathematical model requires at least two partial differential equations, one describing the gas phase and one corresponding to the solid phase. A key difficulty in the process is the long processing times that are typically required. We address here the issue of optimization and show that we can choose appropriate pressure and temperature to minimize these processing times.
\end{abstract}

Mathematics Subject Classification. 76S05.

Received: November 24, 1999.

\section{INTRODUCTION}

A variety of materials are produced by infiltration processes. In these techniques a fluid phase (i.e., a gas or a liquid) is transported into a porous structure, where it then reacts to form a solid product. These methods are particularly important for producing composite materials, where the initial porous perform is composed of the reinforcement phase (i.e., fibers, whiskers, or particles) and infiltration produces the matrix [1,2]. A detailed assessment of the relevant reaction and mass transport rates during infiltration requires mathematical modeling, using a minimum of two coupled partial differential equations which describe changes in the reactant concentration and the solid structure as a function of both position and time. This type of modeling can also be extended to analyze the optimization and control of infiltration processes.

The research presented here specifically considers optimization for a set of two PDE's which describe isothermal, isobaric chemical vapor infiltration (CVI). In this process a vapor-phase precursor is transported into the porous preform, and a combination of gas and surface reactions leads to the deposition of the solid matrix phase. During infiltration the formation of the solid product phase eventually closes off porosity at the external surface of the body, blocking the flow of reactants and effectively ending the process. This is a key feature of most infiltration processes. Isothermal, isobaric CVI often requires extremely long times, so it is generally important to minimize the total processing times.

This paper considers the problem of determining the optimal pressure and temperature which correspond to the minimum infiltration time. From a practical perspective, the nature of the porous preform is often

\footnotetext{
Keywords and phrases. Composites, chemical vapor deposition (CVD), optimization, computer simulation, theory.

* Dedicated to Roger Temam for his 60th birthday

** Research supported by DOE 98ER25346.

1 Division of Applied Mathematics, Brown University Providence, RI 02912, USA. e-mail: dig@cfm.brown.edu

2 Division of Engineering Brown University Providence, RI 02912, USA.
} 
predetermined by the intended application (e.g., the physical dimensions and the fiber size are invariants). Thus, the process can only be controlled with process variables: temperature, pressure, and gas composition. Note that the pressure and temperature determine several different physical quantities in the model, such that the a general understanding of the optimum conditions is not immediately obvious from the basic formulation.

We adopted two strategies to solve the optimization problem. In the first we get an asymptotic approximation, valid when $\alpha^{2}$ (defined in Sect. 2) is small. We then used the explicit form of the asymptotic solution to get a functional of the pressure and temperature that estimates the optimal conditions for the process. In the second approach we get the functional numerically.

This paper is organized as follows: Section 2 presents the basic set of two partial differential equations used to model isothermal, isobaric CVI (including initial and boundary conditions). A definition for a successful process and a discussion on the optimization problem is given. In Section 3 an analysis of the optimization problem is given. The analysis performed is based on asymptotic expansions as well as computations. The results of the analysis are optimal working pressure and temperature. In Section 4 the effects of powder formation in the analysis are included in the analysis; here too the pressure and temperature to minimize the final time are provided. In Section 5 a discussion of the significant of these results is presented.

\section{Formulation}

A mathematical description of infiltration requires one or more partial differential equations which describe the evolution of the matrix (i.e., the solid phase), and one additional partial differential equation for each chemical species in the fluid phase. For a simple pore structure, the continuity equation for species $i$ is

$$
\frac{\partial\left(\varepsilon C_{i}\right)}{\partial t}+\nabla \cdot N_{i}=\sum_{r}^{n_{r}} \nu_{i r} R_{r}
$$

where $t$ is time, $\varepsilon$ is the void fraction of the media, $C_{i}$ and $N_{i}$ are the concentration and the flux of species $i$ respectively, $n_{r}$ is the number of the gaseous species, $\nu_{i r}$ is the stoichiometric coefficients for the $i$ th gaseous species in the $r$ th reaction, and $R_{r}$ represents the volumetric reaction rate of reaction $r$.

The basic partial differential equation(s) which describe reaction and mass-transport in porous media (i.e., the fluid phase) are well-established [3,6]. For example, the Dusty-Gas model [7] is typically used to describe multicomponent diffusion and convection in a porous body.

$$
\frac{N_{i}}{D_{K_{i}}}+\frac{R T}{P} \sum_{j \neq i} \frac{\mathrm{C}_{j} N_{i}-C_{i} N_{j}}{\mathcal{D}_{M_{i j}}}=-\nabla C_{i}-\frac{C_{i} B_{e}}{\mu D_{K_{i}}} \nabla P
$$

where $R$ is the gas constant, $B_{e}$ is the permeability of the media, $\mu$ is the viscosity of the mixture $P$ is the total pressure and $T$ is the temperature. $\mathcal{D}_{M_{i j}}$ and $D_{K_{i}}$ are the effective binary diffusivity for species $i$ and $j$ and the effective Knudsen diffusivity of species $i$, respectively.

In this paper we consider the binary (two gases mixture), diffusion-limited process, with one dilute reactant species in one spatial dimension. Under this conditions $P$ is constant.

Describing the evolution of the matrix phase is equivalent to considering the change in the void fraction, $\varepsilon$ (i.e., the volume fraction of gas inside of the porous solid). The evolution of $\varepsilon$ is given by:

$$
\frac{\partial \varepsilon}{\partial t}=-u S_{v}(\varepsilon)
$$

where $u$ is the rate at which the solid product grows [volume/area/time] and $S_{v}(\varepsilon)$ is the gas/solid surface area per unit volume of the porous solid. 
TABLE 1. Values of the constants.

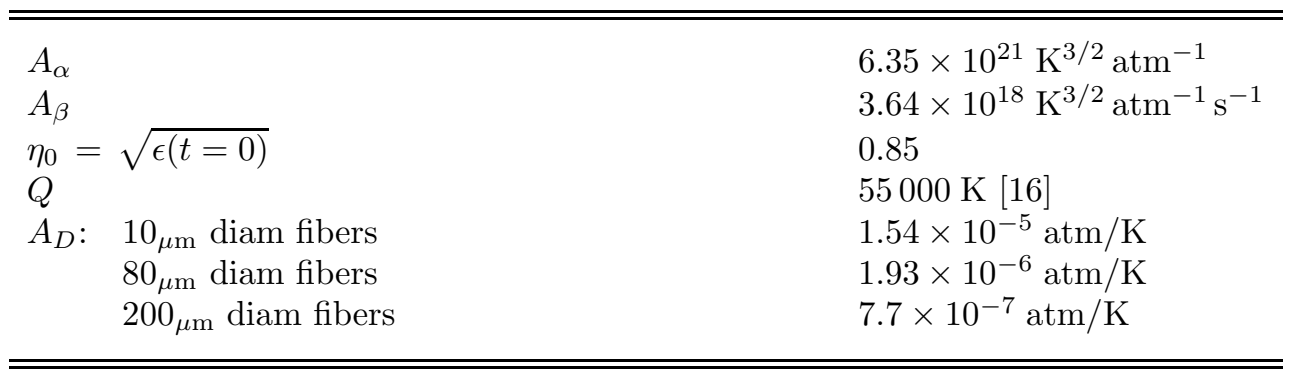

The preforms used for CVI typically have a complex porous structure. However, a cylindrical pore is often used to formulate simple models. This leads to the following expression for $S_{v}$ :

$$
S_{v}(\varepsilon)=\frac{2 \sqrt{\varepsilon_{o}} \sqrt{\varepsilon}}{r_{0}}
$$

where $r_{0}$ is the initial pore radius and $\varepsilon_{o}$ is the initial concentration of $\varepsilon$.

These assumptions leads to the following set of equations, (for more details on the derivation of the model see $[4])$ :

$$
\begin{aligned}
\frac{\partial \eta(z, t)}{\partial t} & =-\frac{\beta(t)}{2} c(z, t) \\
\frac{\partial}{\partial z}\left(f(\eta(z, t)) \frac{\partial c(z, t)}{\partial z}\right) & =\alpha^{2}(t) \eta(z, t) c(z, t)
\end{aligned}
$$

where

$$
\begin{aligned}
z & =\frac{Z}{L} \\
c & =\frac{C(z, t)}{C(0, t)} \\
f(\eta) & =\frac{P \eta^{3}}{3\left(P \eta+A_{D} T\right)} \\
\eta & =\sqrt{\varepsilon} \\
\alpha^{2} & =A_{\alpha} \frac{P e^{(-Q / T)}}{T^{3 / 2}} \\
\beta & =A_{\beta} \frac{X_{0} P e^{(-Q / T)}}{T},
\end{aligned}
$$

where $L$ is the thickness of the preform, $r_{0}$ is the initial pore radius and $X_{0}$ denotes the fraction of the active gas in the inlet (at $z=0) . Q=E / R$, where $E$ is the activation energy. $D_{D}$ is a lumped constant which is proportional to the ratio of the molecular and Knudsen diffusivities. Note that $\alpha^{2}$ is dimensionless and that $\beta$ has units of inverse time. The time derivative in equation (1) has been removed in equation (6), which is permissible because a pseudo steady state $C$ profile is achieved in a short amount of time (i.e., compared to the time scale over which $\varepsilon$ changes) [5]. Transforming $\varepsilon$ to $\eta$ simplifies equation (5). Note that $\eta$ is proportional to $S_{v}$, so $\eta$ can be viewed as a dimensionless surface area per volume. Values of the constants in equations (9-12) are given in Table 1, for the case of carbon CVI from methane, with a preform thickness of $2 L=3 \mathrm{~mm}$. 
The system $(5,6)$ is subject to the initial condition

$$
\eta(z, 0)=\eta_{0}(z)
$$

This paper treats only the case of a uniform initial condition, $\eta_{0}(z)=\eta_{0}$.

The boundary conditions most often used for CVI models are to fix the concentration at the outer surface of the preform, and to assume that diffusion occurs in from two opposite sides, such that there is no net flux in the middle of the preform (i.e., at $Z=L$, where $L$ is the half-thickness of this "virtual" preform). Thus the boundary conditions are:

$$
\begin{gathered}
c(0, t)=1 \\
\left.\frac{\partial c}{\partial z}\right|_{z=1}=0 .
\end{gathered}
$$

With this model it can be shown that:

1. The value of the void function in the inlet $z=0$, is

$$
\eta(0, t)=\eta_{0}-\frac{\beta}{2} t
$$

2. A classical solution exists and is unique for any time $t<t_{c}, t_{c}=\frac{2 \eta_{0}}{\beta}$.

3. At the critical time $t_{c}=\frac{2 \eta_{0}}{\beta}$ the void function $\eta$ vanishes at $z=0$, the inlet closes completely, and the process ends.

4. The void function $\eta$ and the concentration function $c$ are bounded from above and below. $0<c(z, t) \leq 1$, $\eta(0, t) \leq \eta(z, t) \leq \eta_{0}$ for $t<t_{c}=\frac{2 \eta_{0}}{\beta}$.

5. For any time $t<t_{c}$, the void function $\eta(z, t)$ is monotonically increasing function of the spatial variable $z$.

6 . The concentration function $c(z, t)$ is monotonically decreasing function of the spatial variable $z$ and

$$
c(z, t) \leq \frac{\cosh \gamma(1-z)}{\cosh \gamma}
$$

where

$$
\gamma=\sqrt{\frac{\eta_{0}}{f\left(\eta_{0}\right)}} \alpha
$$

The existence and uniqueness were proved by Marion (private communication) the rest of the properties were proven in [12] in Lemme 1 to 6.

A better lower bound on the concentration function $c(z, t)$ is needed for our optimization technique. This will be done in the following theorem:

Theorem 1. Let $c(z, t)$ be the solution of (5) for $t<t_{c}$, then it is bounded from below by

$$
c(z, t) \geq\left[\frac{\cosh \gamma(1-z)}{\cosh \gamma}\right]^{\frac{f\left(\eta_{0}\right)}{f(\eta(0, t))}}
$$

where $\gamma$ is defined in (17). 
Proof. We first note that the function

$$
\frac{\cosh \gamma(1-z)}{\cosh \gamma}
$$

solves the equation

$$
\frac{\partial}{\partial z}\left(f\left(\eta_{0}\right) \frac{\partial w}{\partial z}\right)-\alpha^{2} \eta_{0} w=0
$$

with the boundary conditions $w(0)=1, w^{\prime}(1)=0$. To complete the proof we need the following comparison Lemma:

Lemma 1. Let, for $0 \leq z \leq 1$

$$
\begin{aligned}
& \frac{\partial}{\partial z}\left(a(z) \frac{\partial u}{\partial z}\right)-b(z) u=0 ; \quad 0<b \leq B \\
& \frac{\partial}{\partial z}\left(A(z) \frac{\partial w}{\partial z}\right)-B(z) w=0 ; \quad 0<a \leq A
\end{aligned}
$$

where $a(z), b(z), A(z), B(z)$ are $C^{1}(0,1)$ functions. With boundary conditions:

Then:

$$
\begin{aligned}
u(0) & =w(0)>0, \\
\frac{\partial}{\partial z} u(1) & =\frac{\partial}{\partial z} w(1)=0 .
\end{aligned}
$$

$$
u, w>0, \quad \frac{\partial}{\partial z} u, \frac{\partial}{\partial z} w<0
$$

and

$$
\begin{aligned}
& w(z) \leq w(0) e^{\int_{0}^{z} \frac{a}{A} \frac{u^{\prime}}{u} \mathrm{~d} \zeta} \\
& u(z) \geq u(0) e^{\int_{0}^{z} \frac{A}{a} \frac{w^{\prime}}{w} \mathrm{~d} \zeta} .
\end{aligned}
$$

Proof. We quote Lemma 3 in [12] that shows that $u, w>0$ and $\frac{\partial}{\partial z} u, \frac{\partial}{\partial z} w<0$.

By multiplying equation (20) by $w$, equation (21) by $u$ and subtracting the two equations we get:

$$
w\left(a u^{\prime}\right)^{\prime}-b u w-u\left(A w^{\prime}\right)^{\prime}-B u w=0
$$

which is equivalent to

$$
\left(w a u^{\prime}\right)^{\prime}-\left(u A w^{\prime}\right)^{\prime}=(b-B) u w+(a-A) u^{\prime} w^{\prime} \leq 0 .
$$

Then by integrating between $z$ and 1 and making use of the boundary conditions we get:

$$
w a u^{\prime}-u A w^{\prime} \geq 0 .
$$

we get equations (22) and (23) by solving this inequality. 
From the Lemma it is clear that

$$
u(z) \geq w(z)^{\frac{\max A(z)}{\min a(z)}} .
$$

We are ready now to prove the theorem. We consider equations (6) and (19) and identify

$$
A(z)=f\left(\eta_{0}\right), B(z)=\alpha^{2} \eta_{0}, \quad u=c(z, t),
$$

and

$$
a(z)=f(\eta), b(z)=\alpha^{2} \eta, \quad w=\frac{\cosh (\gamma(1-z))}{\cosh \gamma} .
$$

Since $\eta \leq \eta_{0}$ and $f(\eta(0, t)) \leq f(\eta) \leq f\left(\eta_{0}\right)$ we can apply Lemma 1 to get (18).

The parameters $\alpha^{2}$ and $\beta$ depend on the three key process variables: $T, P$, and $X_{0}$. Process optimization during CVI is achieved by setting these variables to optimal values. In isothermal, isobaric CVI the infiltration kinetics are controlled by diffusion and the deposition reaction. To achieve relatively uniform infiltration, diffusion must be fast relative to the deposition rate. This is typically accomplished by choosing processing conditions that result in a slow deposition rate, which usually leads to long infiltration times. Thus, the primary basis for process optimization is to obtain the desired amount of infiltration in the shortest possible time.

We will thus define what is a successful process:

Definition 1. A process is called successful if for some time $t_{f}<t_{c}$

$$
\begin{aligned}
\eta\left(z_{1}, t_{f}\right) & \leq k_{1} \eta_{0} \\
\eta\left(0, t_{f}\right) & =k_{0} \eta_{0}
\end{aligned}
$$

$k_{0} \ll 1, k_{0}<k_{1}, 0<z_{1} \leq 1$.

Equation (24) states that the final values of the void function $\eta$ should be small in the interval between the inlet and the point $z_{1}$ (note that $\eta(z, t)$ is monotonically increasing function of the spatial coordinate $z$ ). In most problems of interest $z_{1}=1$. Conditions (24) and (25) state that the void function should be uniformly small.

We can determine explicitly the final time, $t_{f}$, in fact from $(16,25)$ :

$$
t_{f}=\left(1-k_{0}\right) \eta_{0} \frac{2}{\beta} .
$$

Note that the time for the process to end decreases as a function of $\beta$ (itself a function of the temperature and pressure, given in (12)).

In the next theorem we will establish the fact that one can always find parameters $\alpha$ and $\beta$ (actually, pressure and temperature that determine $\alpha$ and $\beta$ ) to achieve a successful process, in fact we can state:

Theorem 2. For all $0<k_{0}<k_{1}<1$ there exist temperature and pressure such that there is a successful process.

Proof. Property 1. stated that $\eta(0, t)=\eta_{0}-\frac{\beta}{2} t$, so we can always satisfy (24) for the final time $t_{f}$ defined in (26). The harder part is to show that we can always choose $\alpha$ to satisfy the uniformity condition (25).

By integrating equation (5) we get

$$
\eta\left(z_{1}, t_{f}\right)=\eta_{0}-\int_{0}^{t_{f}} \frac{\beta}{2} c\left(z_{1}, t\right) \mathrm{d} t .
$$


We change variables

$$
\tau=E(t)=\eta_{0}-\frac{\beta}{2} t
$$

and note that $E(0)=\eta_{0}, E\left(t_{f}\right)=k_{0} \eta_{0}, \mathrm{~d} \tau=-\frac{\beta}{2} \mathrm{~d} t$. From Theorem 10 we get:

$$
\begin{aligned}
\eta\left(z_{1}, t_{f}\right) & =\eta_{0}-\int_{k_{0} \eta_{0}}^{\eta_{0}} c\left(z_{1}, \tau\right) \mathrm{d} \tau \\
& \leq \eta_{0}-\int_{k_{0} \eta_{0}}^{\eta_{0}}\left[\frac{\cosh \gamma\left(1-z_{1}\right)}{\cosh \gamma}\right]^{\frac{f\left(\eta_{0}\right)}{f(\eta(0, t))}} \mathrm{d} \tau \\
& =\eta_{0}-\left(1-k_{0}\right) \eta_{0}\left[\frac{\cosh \gamma\left(1-z_{1}\right)}{\cosh \gamma}\right]^{\frac{f\left(\eta_{0}\right)}{f(\eta(0, t))}}
\end{aligned}
$$

For a successful process we demand that $\eta\left(z_{1}, t_{f}\right) \leq k_{1} \eta_{0}$, this will be satisfied if we choose $\alpha$ such that:

$$
\left[\frac{\cosh \gamma\left(1-z_{1}\right)}{\cosh \gamma}\right]^{\frac{f\left(\eta_{0}\right)}{f(\eta(0, t))}} \geq \frac{1-k_{1}}{1-k_{0}}
$$

Since $\frac{1-k_{1}}{1-k_{0}}<1$ we have to find $\alpha$ s.t.

$$
\frac{\cosh \gamma\left(1-z_{1}\right)}{\cosh \gamma} \geq\left[\frac{1-k_{1}}{1-k_{0}}\right]^{\frac{f(\eta(0, t))}{f\left(\eta_{0}\right)}}
$$

$\alpha$ can be made small enough such that the left hand side will be as close to 1 as needed.

The goal of the analysis in the following sections is to find the temperature and pressure that minimize the final time $t_{f}$ for achieving a successful process.

\section{Analysis of the optimization PROBlem}

The final time, $t_{f}$ is given by (26) and it is a decreasing function of $\beta(P, T)$, given in (12). However $\beta$ can not be taken arbitrary large without violating the uniformity condition (24). The optimization problem involves therefore maximizing $\beta(P, T)$ with the constraint $\eta\left(z_{1}, t_{f}\right) \leq k_{1} \eta_{0}$. However the expression for $\eta\left(z_{1}, t_{f}\right)$, equation (27), $t_{f}$ is an integration boundary, which makes it a non-standard optimization problem. In order to overcomethis difficulty we make a change of variable, (28), and use the definition for a successful process, (25), to convert the integral boundaries to constants, as was done in the proof for Theorem 2 . This change of variable converts the optimization problem to a standard one.

In order to express this constraint explicitly in terms of $P$ and $T$ we can use either by asymptotic expansions or by direct numerical solution of the system $(5,6)$. 


\subsection{Asymptotic expansions}

In most of the application the parameter $\alpha^{2}$ is small, and therefore it makes sense to expand the solutions in power of $\alpha^{2}$. The formal expansion t the order of $\alpha^{2}$ is:

$$
\begin{aligned}
c(z, t) & \sim 1-\alpha^{2}\left(z-\frac{z^{2}}{2}\right) \frac{E}{f(E)} \\
\eta(z, t) & \sim E+\alpha^{2}\left(z-\frac{z^{2}}{2}\right) \int_{E}^{\eta_{0}} \frac{\hat{E}}{f(\hat{E})} \mathrm{d} \hat{E} \\
\text { and for } f(\eta) & =\frac{P \eta^{3}}{3\left(P \eta+A_{D} T\right)} \\
& =E(t)+3 \alpha^{2}\left(z-\frac{z^{2}}{2}\right)\left[\log \left(\frac{\eta_{0}}{E(t)}\right)+\frac{A_{D} T}{P}\left(\frac{1}{E}-\frac{1}{\eta_{0}}\right)\right],
\end{aligned}
$$

where, again,

$$
E(t)=\eta_{0}-\frac{\beta}{2} t
$$

In the following theorem we shall prove that the asymptotic expansion is accurate for order $O\left(\alpha^{4}\right)$.

Theorem 3. For all $0 \leq z \leq 1, t \leq t_{f}$ the difference between the exact solution and the asymptotical approximation is $O\left(\alpha^{4}\right)$.

Proof. By integrating (6) between $z$ to 1 , dividing by $f(\eta)$ and integrating between 0 to $z$ one gets

$$
c(z, t)=1-\int_{0}^{z} \frac{1}{f(\eta(\zeta, t))} \int_{\zeta}^{1} \alpha^{2} \eta(\xi, t) c(\xi, t) \mathrm{d} \xi \mathrm{d} \zeta
$$

and for $\eta$ we get, by integrating (5) from 0 to $t$ :

$$
\eta(z, t)=\eta_{0}-\frac{\beta}{2} \int_{0}^{t} c(z, \tau) \mathrm{d} \tau=\eta_{0}-\int_{E}^{\eta_{0}} c(z, e) \mathrm{d} e .
$$

In (32) we use the bounds, $c \leq 1$ that $E \leq \eta \leq \eta_{0}$ to get

$$
c \geq 1-\alpha^{2} \frac{\eta_{0}}{f(E)}\left(z-\frac{z^{2}}{2}\right)
$$

and upon substituting (34) into (33) one gets

$$
\eta \leq E+\alpha^{2} \eta_{0}\left(z-\frac{z^{2}}{2}\right) \int_{E}^{\eta_{0}} \frac{1}{f(e)} \mathrm{d} e .
$$

The bounds (34) and (35) can be further refined. Consider, again, the equality (32), but now we use the estimate for $\eta$ from above (35) and the estimate from below to $c(35)$ to get

$$
c \geq 1-\alpha^{2} \frac{E}{f(E)}\left(z-\frac{z^{2}}{2}\right)-\alpha^{4} \frac{\eta_{0} \int_{E}^{\eta_{0}} \frac{1}{f(e)} \mathrm{d} e}{f(E)}\left(\frac{z}{3}-\frac{z^{3}}{6}+\frac{z^{4}}{24}\right) .
$$


In the same way we can get a better estimate estimate from below in $c$ :

$$
\eta \leq E+\alpha^{2}\left(z-\frac{z^{2}}{2}\right) \int_{E}^{\eta_{0}} \frac{e}{f(e)} d e+\alpha^{4} \eta_{0}\left(\frac{z}{3}-\frac{z^{3}}{6}+\frac{z^{4}}{24}\right) \int_{E}^{\eta_{0}} \frac{\int_{\tilde{E}}^{\eta_{0}} \frac{1}{f(e)} \mathrm{d} e}{f(\tilde{E})} \mathrm{d} \tilde{E} .
$$

To get an upper estimate on $c$ we use estimate (34) together with $\eta \geq E$ and

$$
f(\eta) \geq f\left(E+\alpha^{2} \eta_{0}\left(z-\frac{z^{2}}{2}\right) \int_{E}^{\eta_{0}} \frac{1}{f(e)} \mathrm{d} e\right)
$$

to get

$$
\begin{aligned}
c \leq & 1-\alpha^{2} E \int_{0}^{z} \frac{(1-\zeta)}{f\left(E+\alpha^{2} \eta_{0}\left(\zeta-\frac{\zeta^{2}}{2}\right) \int_{E}^{\eta_{0}} \frac{1}{f(e)} \mathrm{d} e\right)} \mathrm{d} \zeta \\
& +\alpha^{4} E \eta_{0} \int_{0}^{z} \frac{1}{f\left(E+\alpha^{2} \eta_{0}\left(\zeta-\frac{\zeta^{2}}{2}\right) \int_{E}^{\eta_{0}} \frac{1}{f(e)} \mathrm{d} e\right)}\left(\frac{1}{3}-\frac{\zeta^{2}}{2}+\frac{\zeta^{3}}{6}\right) \mathrm{d} \zeta \\
\leq & 1-\alpha^{2} E \int_{0}^{z} \frac{(1-\zeta)}{f\left(E+\alpha^{2} \eta_{0}\left(\zeta-\frac{\zeta^{2}}{2}\right) \int_{E}^{\eta_{0}} \frac{1}{f(e)} \mathrm{d} e\right)} \mathrm{d} \zeta+\alpha^{4} \frac{E \eta_{0}}{f(E)}\left(\frac{z}{3}-\frac{z^{3}}{6}+\frac{z^{4}}{24}\right) .
\end{aligned}
$$

Using the Lipschitz continuity of $1 / f$ we get:

$$
c \leq 1-\alpha^{2} \frac{E}{f(E)}\left(z-\frac{z^{2}}{2}\right)+\alpha^{4} E \eta_{0}\left[L\left(\frac{z^{2}}{2}-\frac{z^{3}}{2}+\frac{z^{4}}{8}\right) \int_{E}^{\eta_{0}} \frac{1}{f(e)} \mathrm{d} e+\frac{1}{f(E)}\left(\frac{z}{3}-\frac{z^{3}}{6}+\frac{z^{4}}{24}\right)\right]
$$

where $L$ is the Lipschitz constant of $1 / f$, and by substituting into equation (33)

$$
\begin{aligned}
\eta \geq & E+\alpha^{2}\left(z-\frac{z^{2}}{2}\right) \int_{E}^{\eta_{0}} \frac{e}{f(e)} \mathrm{d} e \\
& +\alpha^{4} \eta_{0} \int_{E}^{\eta_{0}} \tilde{E}\left[L\left(\frac{z^{2}}{2}-\frac{z^{3}}{2}+\frac{z^{4}}{8}\right) \int_{\tilde{E}}^{\eta_{0}} \frac{1}{f(e)} \mathrm{d} e+\frac{1}{f(\tilde{E})}\left(\frac{z}{3}-\frac{z^{3}}{6}+\frac{z^{4}}{24}\right)\right] \mathrm{d} \tilde{E} .
\end{aligned}
$$

Thus the difference between the exact solution and the asymptotical approximation is $O\left(\alpha^{4}\right)$.

In the next subsection the numerical results and the asymptotic expansions are compared, to demonstrate the validity of the expansions in the range of relevant $\alpha^{2}$.

The asymptotic expansion (30) is used to get an explicit form for the uniformity constraint (24) in terms of the temperature $T$ and the pressure $P$. Substituting (30) into (24) gives:

$$
k_{0} \eta_{0}+3 \alpha^{2}\left(z_{1}-z_{1}^{2} / 2\right)\left[\log \left(\frac{1}{k_{0}}\right)+\frac{A_{D} T}{P \eta_{0}}\left(\frac{1}{k_{0}}-1\right)\right] \leq k_{1} \eta_{0}
$$

Substituting $\alpha^{2}$ from (11) yields the following form for the uniformity constraint

$$
J(P, T) \leq\left(k_{1}-k_{0}\right) \eta_{0}
$$

where, using the explicit form of $f(\eta)$, see (9), we get

$$
J(P, T)=3 A_{\alpha} \frac{P}{T^{3 / 2}} e^{-Q / T}\left(z_{1}-z_{1}^{2} / 2\right)\left[\log \left(\frac{1}{k_{0}}\right)+\frac{A_{D} T}{P \eta_{0}}\left(\frac{1}{k_{0}}-1\right)\right] .
$$


Thus uniformity is assured if

$$
P \leq \frac{B_{0}}{B_{2}} T^{3 / 2} e^{Q / T}-\frac{B_{0}}{B_{2}} T
$$

where

$$
\begin{aligned}
B_{0} & =\frac{\eta_{0}\left(k_{1}-k_{0}\right)}{A_{\alpha} 3\left(z_{1}-z_{1}^{2} / 2\right)} \\
B_{1} & =\frac{A_{D}}{\eta_{0}}\left(\frac{1}{k_{0}}-1\right) \\
B_{2} & =\log \left(\frac{1}{k_{0}}\right) .
\end{aligned}
$$

These results can now be used to approximate the temperature and pressure that minimize the time for a successful process. Recall (see (26)) that the final time $t_{f}$ is inversely proportional to $\beta$ given in (12). The final time $t_{f}$ is therefore minimized if the function

$$
F(P, T)=\frac{P}{T} e^{-Q / T}
$$

is maximized under the uniformity constraint (25). Inspection of (48) shows that in order to maximize $\beta$ we have to take the equality sign in (44). Substituting this into (48), it is easily verified that the following function must be maximize:

$$
G(T)=\frac{B_{0}}{B_{2}} T^{1 / 2}-\frac{B_{1}}{B_{2}} e^{-Q / T} .
$$

This indicates that the final time $t_{f}$ to achieve a successful process is minimized by choosing temperature and pressure satisfying

$$
\begin{aligned}
T^{3 / 2} e^{Q / T} & =\frac{B_{1} Q}{B_{0}} \\
P & =\frac{B_{1}}{B_{2}}(Q-T) .
\end{aligned}
$$

Moreover the minimal final time $t_{f}^{\min }$ is given by

$$
t_{f}^{\min }=\frac{2\left(1-k_{0}\right) \log \left(1 / k_{0}\right)}{k_{1}-k_{0}} \frac{A_{\alpha} Q}{A_{\beta} X_{0}} 3\left(z_{1}-z_{1}^{2} / 2\right) \frac{1}{T^{1 / 2}(Q-T)} .
$$

Where the temperature $T$ is given by (50).

The explicit formulas (50-52) lead to the following observations:

1. The minimum final time, $t_{f}^{\mathrm{min}}$, decreases as $A_{D}$ decreases (i.e. as the molecular diffusion becomes dominant).

2. $t_{f}^{\min }$ decreases as $k_{1}$ increases. This reflects the fact that increasing $k_{1}$ amounts to relaxing the uniformity condition.

3. As $z_{1}$ increases toward $z_{1}=1$, the minimum final time $t_{f}^{\min }$ increases. 

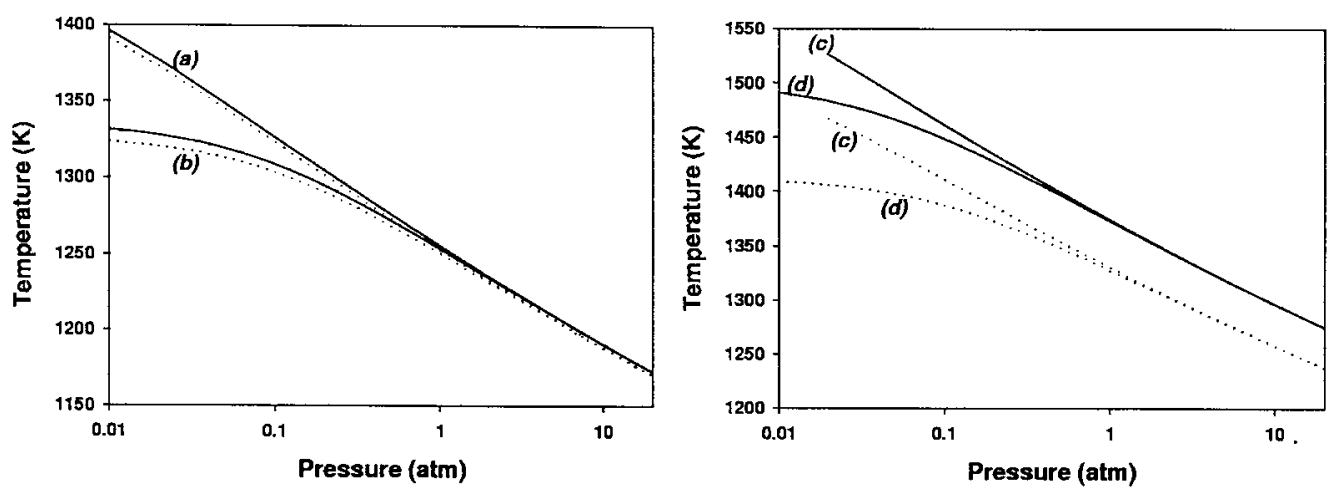

FiguRE 1. J Curves obtained both numerically (solid lines) and by asymptotic analysis (dotted lines). Conditions on or below these lines will satisfy the uniformity condition (Eq. (3.35)). Conditions above this line do not satisfy the uniformity condition. All cases here correspond to $X_{0}=0.1$, with: (a) $k_{1}=0.15, A_{D}=1.54 \times 10^{-5}$; (b) $k_{1}=0.15, A_{D}=7.7 \times 10^{-7}$; (c) $k_{1}=0.7$, $A_{D}=1.54 \times 10^{-5} ;$ (d) $k_{1}=0.7, A_{D}=7.7 \times 10^{-7}$.

\subsection{Computational results}

In the previous subsection the asymptotic expansion of $\eta$ was used to define a functional $J(P, T)$ such that each pair $P, T$ that satisfies $J(P, T) \leq\left(k_{1}-k_{0}\right) \eta_{0}$ leads to a solution that satisfies the conditions for a successful process, $(24,25)$. The optimal $P$ and $T$ was then obtained based on the final time. This result is only approximately correct since the asymptotic expansion was used to approximate condition (24). This section uses numerical solutions of $(5,6)$ to create a "numerical $J$ functional", (i.e. a functional relation between $P$ and $T$ that ensures a successful process).

Two algorithms were used to solve this problem: one based on a finite difference approximation and one on spectral methods. These are described in the Appendix. The schemes were run with $k_{0}=0.1, z_{1}=1$ and $k_{1}=0.15$ or 0.7 . Note that $k_{1}=0.15$ corresponds to relatively uniform infiltration while $k_{1}=0.7$ is relatively non-uniform. Although most applications require relatively uniform infiltration (i.e., lower $k_{1}$ ), there are some cases where a non-uniform profile may be desirable. Two reasons for a higher $k_{1}$ are that it enables faster infiltration times, and it produces materials with lower density. For example, both of these attributes are desirable during the formation of thin carbon-carbon composites for bipolar plates in proton exchange membrane (PEM) fuel cells [17].

Three values were taken for $A_{D}, 1.54 \times 10^{-5}, 1.93 \times 10^{-6}$ and $7.7 \times 10^{-7}$ (see Tab. 1). Plots of the numerical and the asymptotic $J$ curves are presented in Figure 1, plots of $t_{f}$ vs. $P$ are presented in Figure 2.

In Table 2 we present optimal pressure temperature for given $A_{D}$ and $k$.

The process is very sensitive to changes in the temperature $T$, as can be seen, for example, by comparing the results for $P=0.1_{\mathrm{atm}}, T=1309 \mathrm{~K}$, vs. $P=0.1_{\mathrm{atm}}, T=1449 \mathrm{~K}$. Increasing temperature by 140 degrees decreases the final time by a factor of 55 and produces an infiltration profile which is much less uniform. This occurs because of the strong temperature dependence of the deposition reaction. As the fibers size increases $t_{f}$ decreases slightly and the minimal time occurs at lower pressures.

When the uniformity requirement dictates $k_{1}=0.15$ in $(24)$, the condition $J(P, T)=\left(k_{1}-k_{0}\right) \eta_{0}$ yields $P$ and $T$ such that $\alpha^{2} \sim 0.01$. In this case the asymptotic expansions agree well with the numerical results. The predicted temperature to assure uniformity differs by only few degrees from the one obtained numerically, and $t_{f}$ differs by less than $10 \%$. However when $k_{1}$ was increased to 0.7 the asymptotic expansion is less accurate, because, in this case, $\alpha^{2} \sim 0.1$ is not sufficiently small (see Tab. 2). In all cases, however, the asymptotic results agree qualitatively with the numerical results. The curves obtained numerically were almost parallel to the asymptotic, and the points of minima are almost in the same place. The asymptotic results are conservative, 

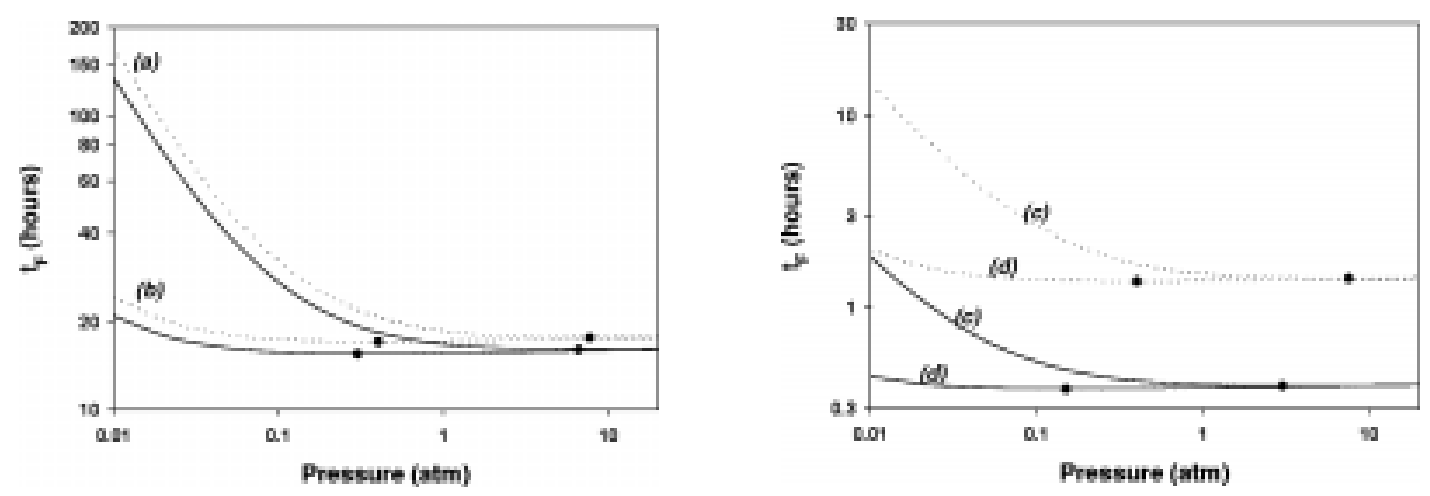

Figure 2. Times which correspond to conditions on the $J$ curves in Figure 1, with values obtained both numerically (solid lines) and by asymptotic analysis (dotted lines). The filled circles show the minimal time. (a) $k_{1}=0.15, A_{D}=1.54 \times 10^{-5}$; (b) $k_{1}=0.15, A_{D}=7.7 \times 10^{-7}$; (c) $k_{1}=0.7, A_{D}=1.54 \times 10^{-5}$; (d) $k_{1}=0.7, A_{D}=7.7 \times 10^{-7}$.

TABLE 2. $t_{f}$ for the optimal $P$ and $T$.

\begin{tabular}{|c|c|c|c|c|c|c|c|}
\hline \multirow[b]{2}{*}{$A_{D}$} & \multirow[b]{2}{*}{$k_{1}$} & \multicolumn{3}{|c|}{ Numerics } & \multicolumn{3}{|c|}{ Asymptotics } \\
\hline & & $P$ & $T$ & $t_{f}$ & $P$ & $T$ & $t_{f}$ \\
\hline $1.54 \times 10^{-5}$ & 0.15 & 6.59817 & 1201.69 & 57703.5 & 7.75487 & 1194.85 & 63436.4 \\
\hline $1.93 \times 10^{-6}$ & 0.15 & 0.83 & 1260.76 & 56363.4 & 0.964282 & 1253.56 & 61971.3 \\
\hline $7.7 \times 10^{-7}$ & 0.15 & 0.334636 & 1288.52 & 55757.3 & 0.373109 & 1282.26 & 61309.3 \\
\hline $1.54 \times 10^{-5}$ & 0.7 & 3.11577 & 1334.74 & 1416.3 & 7.70548 & 1265.71 & 5139.93 \\
\hline $1.93 \times 10^{-6}$ & 0.7 & 0.393241 & 1408.2 & 1379.66 & 0.965 & 1331.69 & 5014.01 \\
\hline $7.7 \times 10^{-7}$ & 0.7 & 0.154896 & 1444.02 & 1363.1 & 0.372904 & 1364.25 & 4957.04 \\
\hline
\end{tabular}

they always overestimated the final time, and gave more restrictive conditions on $P$ and $T$ for uniformity $i . e$. $P$ and $T$ obtained by the asymptotic analysis never predict a successful process if it does not exists. However, since $P$ and $T$ obtained by the asymptotic analysis may be much different from the optimal ones (obtained by the numerical analysis), $t_{f}$ may be much larger then the minimal value (See Tab. 2).

\section{Homogeneous NuCleation}

CVI processes can be limited by homogeneous nucleation (i.e., powder formation) in the gas phase. This effect has not been treated in previous CVI models because it generally occurs outside of the solid preform. However, powder formation can impose serious limitations on CVI operating conditions during the formation of carbon and oxide matrices. Thus, this phenomena imposes a constraint on the allowable CVI operating conditions. In practice, the nucleation rate depends on the reactor configuration, as well as its actual value. For the current analysis, we assume that powder formation limits CVI when the nucleation rate exceeds some allowable level, $I_{\text {lim. }}$. With this in mind, the following constraint can be presented. (For the derivation on this 


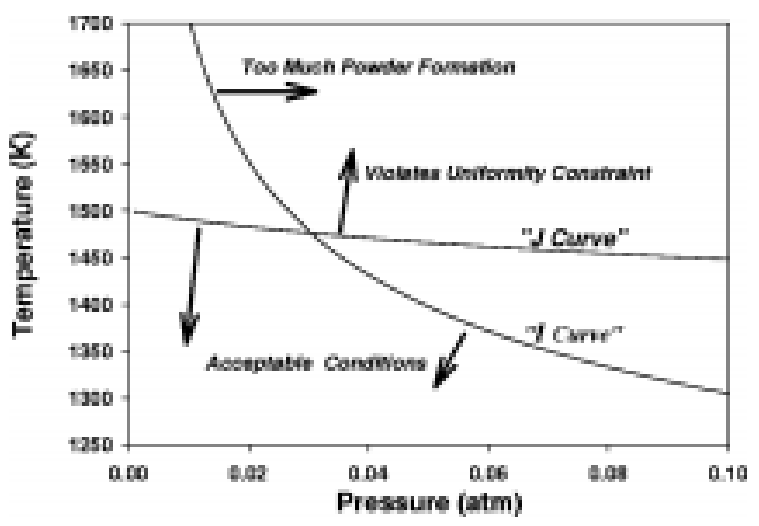

a)

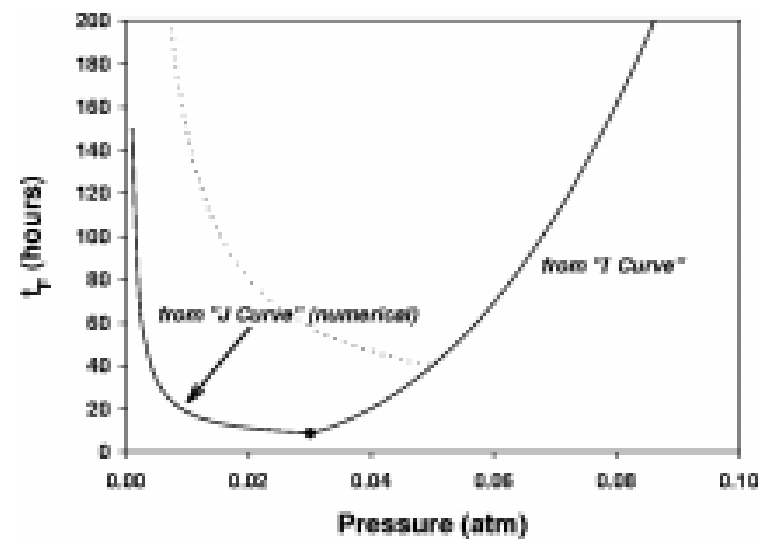

b)

FiguRE 3. Effect of homogeneous nucleation for $k_{1}=0.7, X_{0}=0.01, A_{D}=1.54 \times 10^{-5}$; (a) The numerically obtained $J$ curve (uniformity constraint, Eq. (42)) and the $I$ curve (nucleation limit, Eq. (53) with $A_{\text {lim }}=2.0 \times 10^{21}$ ). (b) Limiting time as a function of pressure. The left part of the curve is determined by the numerically obtained $J$ curve and the right side is determined by the $I$ curve, with the minimal time shown by the filled circle. The dotted line corresponds to the approximate $J$ curve which was determined with asymptotics.

constraint see [4].)

$$
A_{\lim }=\left(X_{0} P\right)^{2} / T^{2.5} \exp \left(-A_{I} / T^{m}\right)
$$

where $A_{\text {lim }}$ is the maximal nucleation rate specification and the two constants, $A_{I}$ and $m$ are determined empirically to fit the nucleation model. A good fit to the experimental data of Loll et al., see [13,14], was obtained with $m=1.5, A_{I}=750000 \mathrm{~K}^{m}$, and $A_{\text {lim }}=3.3 \times 10^{-17} \mathrm{~atm}^{2} / \mathrm{K}^{2.5}$. The value of $A_{\text {lim }}$, however, is somewhat arbitrary, since it reflects a threshold for a given reactor. By varying $A_{\text {lim }}$, it is possible to assess different tolerance levels for powder formation. For example, recent carbon CVI experiments at Oak Ridge National Laboratory tolerate higher powder formation levels than those described by Loll et al. with a threshold value that corresponds to $A_{\text {lim }}=2.0 \times 10^{-21} \mathrm{~atm}^{2} / \mathrm{K}^{2.5}[15]$.

The effect of adding the powder formation constraint can be seen from Figure 3, where the $I$-curves are defined by equation (53). As seen from the left plot, the new constraint limits the pressures and temperatures to values which are below both the $I$ and the $J$-curves. For a given pressure, the minimal $t_{f}$ corresponds to a temperature on either the $I$ or $J$-curve (whichever is lower). Thus if the minimal $t_{f}$ found in Section 3 (i.e. when $P$ and $T$ are on the $J$-curves) to the left of the $I$-curve, then the additional constraint does not change the previous results. If, on the other hand, this point is on the right of the $I$-curve then, the minimal $t_{f}$ occurs at the intersection between the $I$ and $J$-curves. This point can be clearly seen as a cusp in the $t_{f} v s$. $P$ in the right plot.

A complete assessment of $X_{0}$ effects requires solutions with the full Dusty Gas model, because large values of $X_{0}$ violate the assumption of a dilute reactant gas. However, considering only values up to $X_{0}=0.1$ provides useful insight into optimizing dilute systems. Without the homogeneous nucleation constraint (i.e., as $\left.A_{\text {lim }} \rightarrow \infty\right)$, the minimal time is inversely proportional to $X_{0}$, and the optimal pressure and temperature do not vary with $X_{0}$ (see Sect. 3). However, homogeneous nucleation limits the operating conditions when $A_{\text {lim }}$ is low enough, as illustrated in Figure 3. The effect of this limitation on the optimal conditions and on the 


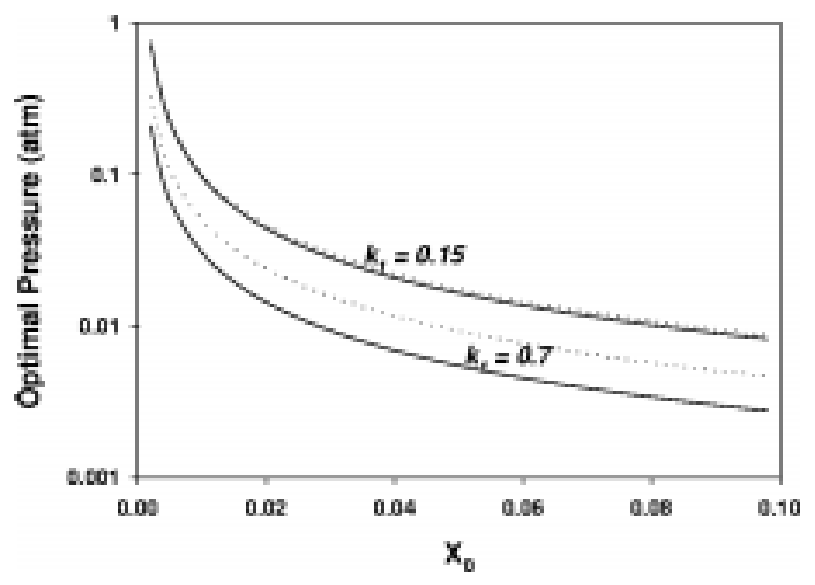

a)

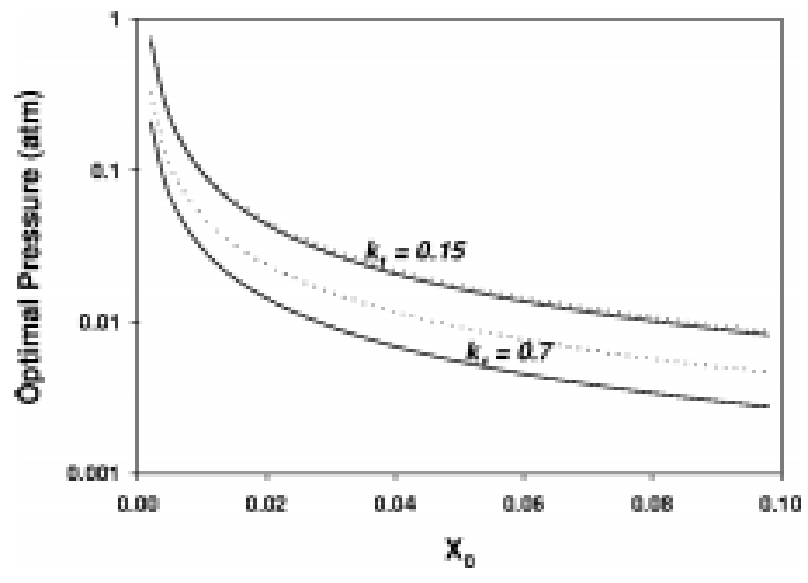

b)

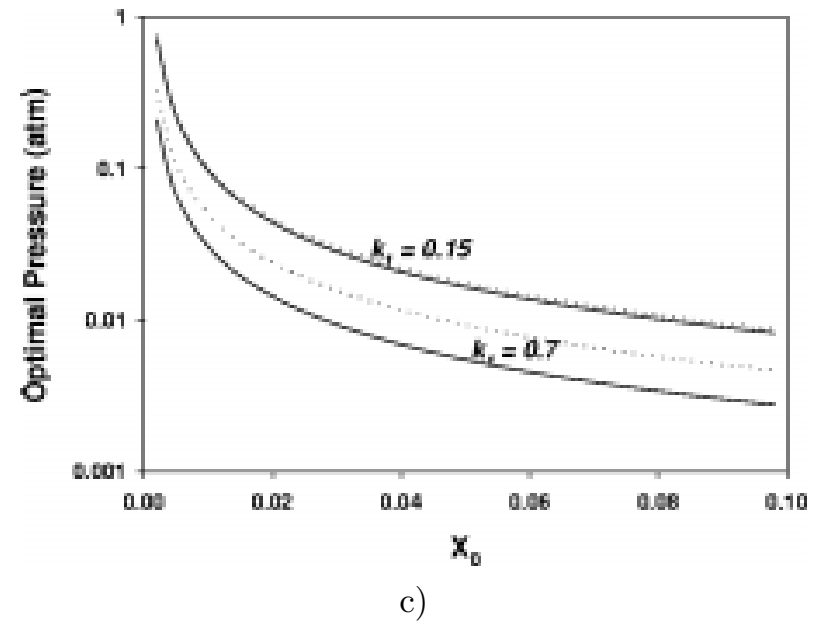

FiguRE 4. Effect of $X_{0}$ with $A_{D}=7.7 \times 10^{-7}$ : (a) optimal pressures; (b) optimal temperatures; (c) minimum infiltration times.

minimal time are shown in Figure 4. These results lead to the following conclusions:

1. As in Section 3, the asymptotic results are in good agreement with the numerical results for $k_{1}=0.15$, where $\alpha^{2} \sim 0.01$, and much less accuracy in the case $k_{1}=0.7$ where $\alpha^{2} \sim 0.1$. In both cases, however, the asymptotic results agree qualitatively with the numerical results.

2. Notwithstanding the dilute reactant gas restriction, $t_{f}$ are monotonically decreasing functions of $X_{0}$, thus it is advisable to work in the "highest" $X_{0}$ possible. However since the optimal $P$ is also a monotonically decreasing functions of $X_{0}$, this value of $X_{0}$ is limited by the lowest operational pressure. For example for working pressure of about $0.01_{\mathrm{atm}}$, the maximum allowable $X_{0}$ is only 0.05 , for $200_{\mu \mathrm{m}}$ diameter fibers $\left(A_{D}=7.7 \times 10^{-7}\right)$.

3. For a given $X_{0}$, as the fiber diameter increases $P$ and $t_{f}$ decrease and $T$ increases. But unlike Section 3, the differences here are significant. This occurs because the homogeneous nucleation condition forces us to work in a region where the dependence on $A_{D}$ is much stronger.

4. The homogeneous nucleation constraint causes the optimal temperature and pressure to vary with $X_{0}$.

Note that the minimum infiltration time is dramatically increased when there is a significant limitation imposed by homogeneous nucleation. In general, the process must be operated at lower pressures to avoid 
powder formation. Some increase in the corresponding optimal temperature accompanies this decrease in pressure.

\section{Conclusions}

Minimizing infiltration times for isothermal, isobaric CVI is important because processing times are typically very long. The work presented here provides a detailed assessment of the pressure and temperature which will minimize the total required time, based on a simplified model for a single, dilute reactant species. This formulation makes it possible to understand the basic physics of the problem in terms of a relatively small number of lumped parameters. The basic objective of this optimization problem is to obtain a density profile with a prescribed amount of uniformity, in the shortest possible time (Sect. 3). The asymptotic results are particularly useful, because they make it possible to determine optimal conditions without doing numerical calculations (under conditions where $\alpha^{2}$ is small enough). Based on comparisons with the numerical results, the asymptotic forms are also qualitatively accurate when $\alpha^{2}$ is larger. Thus, the asymptotic results provide a clear understanding of how the optimal conditions are related to the key parameters for the problem. The effects of homogeneous nucleation were also analyzed, as an additional constraint on the basic optimization problem. This issue has not been considered in previous work on CVI modeling, however, it can limit operating conditions in systems were powder formation is significant (e.g., the formation of carbon matrix composites). The results obtained here provide a quantitative assessment of the conditions where homogeneous nucleation imposes limitations on infiltration conditions. When these limitations occur, powder formation also increases the minimum infiltration time.

\section{REFERENCES}

[1] E. Fitzer and R. Gadow, Am. Ceram. Soc. Bull 65 (1986) 326-355.

[2] S.M. Gupte and J.A. Tsamopoulos, J. Electrochem. Soc. 136 (1989) 555-561.

[3] R. Aris, The Mathematical Theory of the Diffusion and Reaction in Permeable Catalysts, Oxford University Press, London (1975).

[4] A. Ditkowski, D. Gottlieb and B.W. Sheldon, Optimization of Chemical Vapor Infiltration with Simultaneous Powder Formation. J. Mater. Res. (submitted).

[5] H.-C. Chang, Minimizing Infiltration Time during Isothermal Chemical Vapor Infiltration, Ph.D. thesis, Brown University (1995).

[6] F.A.L. Dullien, Porous Media: Fluid Transport and Pore Structure, Academic Press, New York (1979).

[7] E.A. Mason and A.P. Malinauskas, Gas Transport in Porous Media: The Dusty-Gas Model, Elsevier Science Publisher (1983).

[8] B.W. Sheldon and H.-C. Chang, in Ceramic Transactions, Vol. 42, B.W. Sheldon and S.C. Danforth Eds (American Ceramic Society) (1994) 81-93.

[9] H.-C. Chang, T.F. Morse and B.W. Sheldon, J. Mater. Proc. Manuf. Sci. 2 (1994) 437-454.

[10] J.Y. Ofori and S.V. Sotirchos, AIChE Journal 42 (1996) 2828.

[11] H.-C. Chang, T.F. Morse and B.W. Sheldon, J. Am. Ceram. Soc. 7 (1997) 1805-1811.

[12] H.-C. Chang, D. Gottlieb, M. Marion and B.W. Sheldon, J. of Scientific Computing 13 (1998) $303-321$.

[13] P. Loll, P. Delhaes, A. Pacault and A. Pierre, Carbon 13 (1975) 159.

[14] P. Delhaes, in Electrochemical Society Proceedings 97-25, M.D. Allendorf and C. Bernard Eds (Electrochemical Society) (1997) 486-495.

[15] T.M. Besmann, Oak Ridge National Laboratory, unpublished results (1998).

[16] S. Bammidipati, G.D. Stewart, G.R. Elliott Jr, S.A. Gokoglu and M.J. Purdy, AIChE Journal 42, No. 11, (1996) 3123-3132.

[17] T.M. Besmann, J.W. Klett and T.D. Burchell, in MRS Symposium Proceedings (Materials Research Society, Pittsburgh, 1998) $365-370$. 